\title{
Correction to Clinical anatomy of the lumbar sinuvertebral nerve with regard to discogenic low back pain and review of literature
}

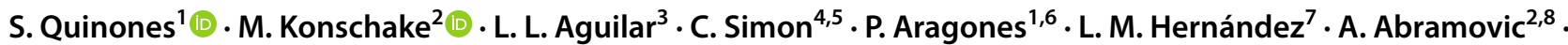 \\ S. R. Tubbs ${ }^{9,10,11,12,13,14,15}$. Jose Bouzada ${ }^{1} \cdot$ F. J. Valderrama-Canales ${ }^{1} \cdot$ T. Vazquez $^{1} \cdot$ J. Sanudo ${ }^{1}$ (1)
}

Published online: 18 June 2021

○) Springer-Verlag GmbH Germany, part of Springer Nature 2021

Correction to: European Spine Journal https://doi.org/10.1007/s00586-021-06886-1

Unfortunately, the 9th author name was incorrectly published in the original publication. The complete correct name should read as follows.

Jose Bouzada

The original article has been corrected.

Publisher's Note Springer Nature remains neutral with regard to jurisdictional claims in published maps and institutional affiliations.

The original article can be found online at https://doi.org/10.1007/ s00586-021-06886-1.

M. Konschake

marko.konschake@i-med.ac.at

1 Department of Anatomy and Embryology, Faculty of Medicine, Universidad Complutense de Madrid, Madrid, Spain

2 Department of Anatomy, Histology and Embryology, Institute of Clinical and Functional Anatomy, Medical University of Innsbruck (MUI), Müllerstr. 59, 6020 Innsbruck, Austria

3 Unidad de Columna. Servicio de COT., Hospital Plató., Barcelona, Spain

4 Computer Science and Statistics, URJC., Madrid, Spain

5 Instituto Universitario de Evaluación Sanitaria, UCM, Madrid, Spain

6 Department of Orthopedics. Hospital, Universitario Santa Cristina, Madrid, Spain

7 Human Anatomy and Embryology Unit, Department of Surgery, Medical and Social Sciences, Scholl of Medicine and Health Sciences, University of Alcalá, UAH, Alcalá de Henares, Madrid, Spain

8 Department of Neurosurgery, Medical University of Innsbruck (MUI), Innsbruck, Austria

9 Department of Neurosurgery, Tulane University School of Medicine, New Orleans, LA, USA

10 Department of Neurology, Tulane University School of Medicine, New Orleans, LA, USA

11 Department of Surgery, Tulane University School of Medicine, New Orleans, LA, USA

12 Department of Structural and Cellular Biology, Tulane University School of Medicine, New Orleans, LA, USA

13 Department of Neurosurgery and Ochsner Neuroscience Institute, Ochsner Health System, New Orleans, LA, USA

14 Department of Anatomical Sciences, School of Medicine, St. George's University, West Indies, Grenada

15 University of Queensland, Brisbane, Australia 